\title{
Repair Responses of Pulp Tissues after Different Direct Pulp Capping Materials at Different Storage Time
}

\author{
Ali Yakout Dogheim, Khaled Mohamed Noaman, Khadiga Youssef Kawana, \\ and Walaa Mohamed Al Samolly
}

\section{ABSTRACT}

Objective: The objective of this study was to evaluate and compare histologically the repair response following direct pulp capping of mechanically exposed dogs' teeth with: Dycal, UltraBlend Plus and TheraCal LC: at different time intervals (1 week, 1 month and 3 months)

Materials and methods: A total of 54 permanent teeth of six healthy male mongrel dogs were used, divided into three groups $(n=18)$ according to pulp capping material used. Group I: Dycal, Group II: UltraBlend Plus and Group III: TheraCal LC. All cavities were restored with Riva LC Resine Modified Glass Ionomer. Each group was subdivided into three subgroups according to storage time $(n=6)$. Control samples ( 3 teeth) were added representing normal untouched teeth. The specimens were fixed, decalcified, processed to paraffin blocks. Serial sections of 5 microns thickness were prepared and stained with $H \& E$ then examined under light microscope for histologic examination and were evaluated histologically for pulp tissue organization and reparative tissue formation following scoring system used by Nowicka et al.

Results: As regards calcific barrier, all three materials, by the end of third month showed: continuity (score 3) with irregular dentin bridge formation, the morphology showed mostly irregular hard tissue and the tubules were mild in most specimens.

Conclusion: Theracal is superior to Dycal and UBP for pulp capping of mechanically exposed human teeth, it can be used as an effective direct pulp capping material. There was no statistically significant difference between the three capping agents by end of third evaluation period as regards calcific barrier and odontoblastic layer.

Keywords: Calcific barrier, Direct pulp capping, Dycal, TheraCal, Ulrtra blend plus.

\section{INTRODUCTION}

As pulp capping materials are in direct contact with pulp, the cytotoxicity and biocompatibility are of particular importance in order to avoid pulp irritation and maintain pulp vitality [1].

Many factors influence pulp capping: the cause of vital pulp exposure, the age of the pulp, the size and time of pulp exposure, bleeding control and disinfection [2]-[4].

Many studies indicates that calcium hydroxide compounds are the gold standard for pulp capping [5]. As a result of its limitations different formulations of $\mathrm{Ca}(\mathrm{OH})_{2}$ have been developed and several dental adhesives have been proposed in direct pulp capping. Hebling J et al [6] in 2009 evaluated the cytotoxic effects of resin-based light-cured liners like TheraCal and Ultrablend Plus (UBP), they concluded that Theracal was least cytotoxic when compared to other materials. Chaudhari WA et al in 2016 compared calcium (Ca) ion-releasing capacity of four different lightcured calcium hydroxide $\left[\mathrm{Ca}(\mathrm{OH})_{2}\right]$ cements with self-cured $\mathrm{Ca}(\mathrm{OH})_{2}$ cement, Septocal, TheraCal, Cal LC and they
Published Online: May 27, 2021

ISSN: $2684-4443$

DOI: $10.24018 /$ ejdent.2021.2.3.55

Ali Yakout Dogheim*

Specialist, Conservative Department, Faculty of Dental Medicine, Alexandria University, Egypt.

(e-mail: ali_yakout@hotmail.com)

Khaled Mohamed Noaman

Professor, Department of Operative Dentistry, Faculty of Dental Medicine, Boys, Cairo, Al-Azhar University, Egypt. (e-mail: khalidnoaman ${ }^{\circledR}$ yahoo.com) Khadiga Youssef Kawana

Professor, Department of Oral Biology, Faculty of Dental Medicine, Alexandria University, Egypt.

(e-mail: khadigakawana ${ }^{@}$ yahoo.com)

Walaa Mohamed Al Samolly

Lecturer, Department of Operative Dentistry, Faculty of Dental Medicine, Boys, Cairo, Al-Azhar University, Egypt. (e-mail: walaasamolly@gmail.com)

*Corresponding Author concluded that Hydrocal and TheraCal were found to be the highest light-cured Calcium ion releasing materials [7].

As the choice between the wide variety of pulp capping material is somewhat difficult, the objective of this study was to evaluate and compare the histologic repair response at different time intervals ( 1 week, 1 month and 3 months) following direct pulp capping of mechanically exposed dogs' teeth with: Dycal, UltraBlend Plus and TheraCal LC.

\section{MATERIALS AND METHODS}

A total of 54 permanent teeth of six healthy male mongrel dogs aged 18-24 months and weighing about 18-24 kg were used in this study.

The selected teeth were Canine, second, third and fourth premolars and, first molar, they were free from caries, fractures, and periodontal disease, they were randomly divided into three groups $(n=18)$ according to pulp capping material used. Group I: Dycal, Group II: UltraBlend Plus and Group III: TheraCal LC. All cavities were restored with Riva LC Resine Modified Glass Ionomer. Each group was subdivided into three subgroups according to storage time 
$(n=6)$ at time intervals of 1 week, 1 month and 3 months Three control teeth untouched were used to evaluate and compare the studied group with normal pulp histology.

Operations were done under general anesthesia with intramuscular injection of $10 \mathrm{mg} / \mathrm{kg}$ of Ketamine $\mathrm{HCl}$ and $1 \mathrm{mg} / \mathrm{kg}$ of Xylazine. The operation field, dogs' teeth and oral cavity were cleaned and disinfected with $0.2 \%$ Chlorhexidine and rubber dam isolation was performed.

A class $\mathrm{V}$ cavity was prepared $1 \mathrm{~mm}$ away from gingival margin on the buccal surface of the teeth until pinkish shadow of the pulp was seen using a bur number 330 in high-speed hand piece with copious water spray. A new bur was used for every four cavities to avoid heat generation. A number one sized round carbide bur was used to drill in the middle of the cavity to expose the pulp in a diameter not exceeding one mm. Bleeding was controlled using sterile cotton pellets soaked in saline for few seconds before placing pulp capping materials. Pulp capping materials were applied according to manufacturer instructions Dycal was mixed in ratio 1:1 base paste to catalyst paste and placed by burnisher in the cavity not more than $1 \mathrm{~mm}$ in thickness and left 2-6 min, UltraBlend Plus was injected in the cavity at the exposure site and light cured for 20 seconds and Theracal was injected in the cavity at the exposure site and light cured for 20 seconds. All materials were placed into the exposed pulp without pushing it into the pulp tissue, materials were randomly assigned into each quadrant.

Two dogs were sacrificed with intravenous anesthetic overdose by injection of concentrated thiopental at the interval 1 week, 1 month and 3 months.

Jaw segments including the operated teeth and parts of surrounding soft tissue were cut out of the animal jaw. The specimens were fixed in $10 \%$ prepared neural buffered formalin, decalcified, and processed to paraffin blocks. Serial sections of 5 microns thickness were prepared and stained with $\mathrm{H} \& \mathrm{E}$ then examined under light microscope for histologic examination.

The specimens were examined and evaluated histologically for calcific barrier continuity, morphology, presence of tubules and odontoblast cells evaluation following scoring system used by Nowicka et al [8] (Table I).

TABLE I: SCORING SYSTEM [8]

\footnotetext{
Scores Calcific barrier continuity

1. Complete dentin bridge formation.

2. Partial/incomplete dentin bridge formation extending to more than one-half

of the exposure site but not completely closing the exposure site.

3. Initial dentin bridge formation extending to not more than one-half of the exposure site.

4. No dentin bridge formation.

Scores Calcific barrier morphology

1. Dentin or dentin associated with irregular hard tissue.

2. Only irregular hard tissue deposition.

3 . Only a thin layer of hard tissue deposition.

4. No hard tissue deposition.

Scores Tubules in calcific barrier

1. No tubules present.

2. Mild (tubules present in $<30 \%$ of calcific barrier).

3. Moderate to severe (tubules present in $>30 \%$ of calcific barrier).

Scores Odontoblastic cell layer

1. Palisade pattern of cells.

2. Presence of odontoblast cells and odontoblast-like cells.

3. Presence of odontoblast-like cells only.

4. Absent.
}

\section{RESULTS}

A. Comparison of the Effect of the Three Capping Materials on Calcific Barrier Score as Function of Evaluation Periods

At one week interval: As regards dentine barrier continuity all cases of 1 week period $100 \%$ showed no dentine bridge formation in all three materials used, the morphology in all cases showed no hard tissue deposition in $100 \%$ of cases and the tubules were absent in $100 \%$ of cases. No statistically significant difference was detected between the three materials after one week as regards odontoblastic cell layer.

At one month: No statistically significant difference was present between the three materials after one month as regards calcific barrier and odontoblastic cell layer

At three months: No statistically significant difference was seen as regards calcific barrier and odontoblastic cell layer between the three materials.

B. Comparison between the Effect of Each Capping Material on Calcific Barrier Score and Odontoblastic Layer at Different Evaluation Periods

For Dycal the dentine barrier continuity, the morphology with irregular hard tissue deposition and the presence of tubules were statistically significant between the three intervals while no statistically significant difference was detected at different time intervals as regards odontoblastic cell layer.

For UBP the results were statistically significant concerning calcific barrier continuity, morphology as well as presence of tubules. No statistically significant difference was detected at different time intervals as regards odontoblastic cell layer.

For theracal the calcific barrier continuity, morphology as well as tubules results were statistically significant between the three studied periods.

No statistically significant difference was detected at different time intervals as regards odontoblastic cell layer.

Regarding the effect of capping material on calcific barrier score as function of evaluation periods, the data were collected and tabulated in Table II.

\section{Histological Evaluation Results}

Histological evaluation was based on examination and evaluation of pulp reaction to different dental materials according to modified scoring system adapted after Nowicka et al [8].

\section{Normal Pulp (Control Group) (Fig. 1)}

The histological sections showed normal histological features of pulp tissues.

1. First observation period (one week interval) (Fig. 2)

The superficial area directly opposite to the exposure site showed severe destruction of the odontoblastic layer, with loss of the normal architecture of the connective tissue stroma in Dycal and UBP groups. For TheraCal group: the odontoblastic layer showed disorganization although the outline of the odontoblast could be detected. 
TABLE II: PREVALENCE AND TEST OF SigNIFICANCE FOR THE EFFECT OF CAPPING MATERIAL ON CALCIFIC BARRIER SCORE AS FuNCTION OF EVALUATION PERIODS

\begin{tabular}{|c|c|c|c|c|c|c|c|c|c|c|c|c|c|c|c|c|c|c|}
\hline \multirow{3}{*}{ Calcific barriers } & \multicolumn{6}{|c|}{1 week } & \multicolumn{6}{|c|}{1 month } & \multicolumn{6}{|c|}{3 month } \\
\hline & \multicolumn{2}{|c|}{$\begin{array}{l}\text { Dycal } \\
(\mathrm{n}=6)\end{array}$} & \multicolumn{2}{|c|}{$\begin{array}{l}\text { Ultra blend } \\
\text { plus }(n=6)\end{array}$} & \multicolumn{2}{|c|}{$\begin{array}{c}\text { Theracal } \\
(n=6)\end{array}$} & \multicolumn{2}{|c|}{$\begin{array}{l}\text { Dycal } \\
(\mathrm{n}=6)\end{array}$} & \multicolumn{2}{|c|}{$\begin{array}{l}\text { Ultra blend } \\
\text { plus }(n=6)\end{array}$} & \multicolumn{2}{|c|}{$\begin{array}{c}\text { Theracal } \\
(\mathrm{n}=6)\end{array}$} & \multicolumn{2}{|c|}{$\begin{array}{l}\text { Dycal } \\
(\mathrm{n}=6)\end{array}$} & \multicolumn{2}{|c|}{$\begin{array}{l}\text { Ultra blend } \\
\text { plus }(n=6)\end{array}$} & \multicolumn{2}{|c|}{$\begin{array}{c}\text { Theracal } \\
(n=6)\end{array}$} \\
\hline & No. & $\%$ & No. & $\%$ & No. & $\%$ & No. & $\%$ & No. & $\%$ & No. & $\%$ & No. & $\%$ & No. & $\%$ & No. & $\%$ \\
\hline \multicolumn{19}{|l|}{ Continuity } \\
\hline 1 & 0 & 0.0 & 0 & 0.0 & 0 & 0.0 & 0 & 0.0 & 0 & 0.0 & 1 & 16.7 & 1 & 16.7 & 1 & 16.7 & 2 & 33.3 \\
\hline 2 & 0 & 0.0 & 0 & 0.0 & 0 & 0.0 & 1 & 16.7 & 1 & 16.7 & 4 & 66.7 & 5 & 83.3 & 3 & 50.0 & 4 & 66.7 \\
\hline 3 & 0 & 0.0 & 0 & 0.0 & 0 & 0.0 & 4 & 66.7 & 5 & 83.3 & 1 & 16.7 & 0 & 0.0 & 2 & 33.3 & 0 & 0.0 \\
\hline 4 & 6 & 100 & 6 & 100 & 6 & 100 & 1 & 16.7 & 0 & 0.0 & 0 & 0.0 & 0 & 0.0 & 0 & 0.0 & 0 & 0.0 \\
\hline${ }^{\mathrm{MC}} \mathrm{p}$ & \multicolumn{6}{|c|}{-} & \multicolumn{6}{|c|}{0.107} & \multicolumn{6}{|c|}{0.621} \\
\hline \multicolumn{19}{|l|}{ Morphology } \\
\hline 1 & 0 & 0.0 & 0 & 0.0 & 0 & 0.0 & 2 & 33.3 & 1 & 16.7 & 1 & 16.7 & 1 & 16.7 & 1 & 16.7 & 2 & 33.3 \\
\hline 2 & 0 & 0.0 & 0 & 0.0 & 0 & 0.0 & 4 & 66.7 & 4 & 66.7 & 4 & 66.7 & 4 & 66.7 & 5 & 83.3 & 4 & 66.7 \\
\hline 3 & 0 & 0.0 & 0 & 0.0 & 0 & 0.0 & 0 & 0.0 & 1 & 16.7 & 1 & 16.7 & 1 & 16.7 & 0 & 0.0 & 0 & 0.0 \\
\hline 4 & 6 & 100 & 6 & 100 & 6 & 100 & 0 & 0.0 & 0 & 0.0 & 0 & 0.0 & 0 & 0.0 & 0 & 0.0 & 0 & 0.0 \\
\hline${ }^{\mathrm{MC}} \mathrm{p}$ & \multicolumn{6}{|c|}{-} & \multicolumn{6}{|c|}{1.000} & \multicolumn{6}{|c|}{1.000} \\
\hline \multicolumn{19}{|l|}{ Tubules } \\
\hline 1 & 6 & 100 & 6 & 100 & 6 & 100 & 0 & 0.0 & 0 & 0.0 & 0 & 0.0 & 0 & 0.0 & 0 & 0.0 & 0 & 0.0 \\
\hline 2 & 0 & 0.0 & 0 & 0.0 & 0 & 0.0 & 4 & 66.7 & 4 & 66.7 & 3 & 50.0 & 2 & 33.3 & 2 & 33.3 & 2 & 33.3 \\
\hline 3 & 0 & 0.0 & 0 & 0.0 & 0 & 0.0 & 2 & 33.3 & 2 & 33.3 & 3 & 50.0 & 4 & 66.7 & 4 & 66.7 & 4 & 66.7 \\
\hline${ }^{\mathrm{MC}} \mathrm{p}$ & \multicolumn{6}{|c|}{-} & \multicolumn{6}{|c|}{1.000} & \multicolumn{6}{|c|}{1.000} \\
\hline
\end{tabular}

${ }^{\mathrm{MC}} \mathrm{p}$ : $\mathrm{p}$ value for Chi square test (Monte Carlo) for comparing between the studied groups.

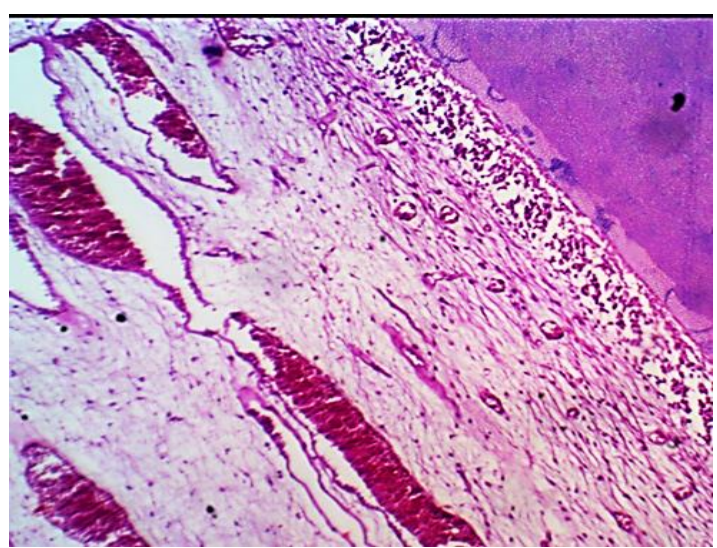

Fig. 1. L.M. Control group showing; normal histological features of the pulp tissue (H\&E X 100).

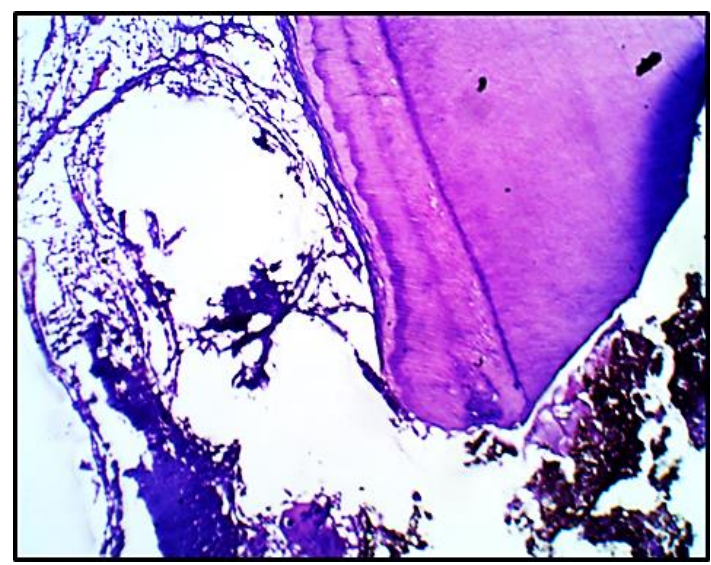

Fig. 2. L.M. Dycal group one week showing; destruction of the odontoblastic layer at the exposure site (H\&E X100).

\section{Second observation period (One month interval) (Fig. $3,4)$}

Dycal group: The tissue formation consisted of a dense ribbon of differentiating odontoblast like cells with vertical thin sheets of collagen fibers which extended towards the inner aspect of the exposure site.

Ultra Blend Plus group: Directly opposite to the exposure site a very thin interrupted hard tissue bridge was seen, the direct transition from necrotic inflammatory odontoblastic layer to multi-layer of vital odontoblasts with congested dilated blood vessels was detected. The predentin was laid down in the exposure site. The outer boundary of the exposure site was limited with flattened odontoblast like cells beneath which scattered fine fibrils were seen extending towards the inner boundary.

TheraCal group: The specimens showed a slight disorganization of the pulp beneath the cavity. Dilated congested blood vessels and very thin irregular bridge of dentin showing osteodentin with some osteocytes were seen in the superficial area directly opposite to the exposure site. The pulp tissue revealed some calcification in thin fibrous connective tissue and multiple layers of odontoblasts.

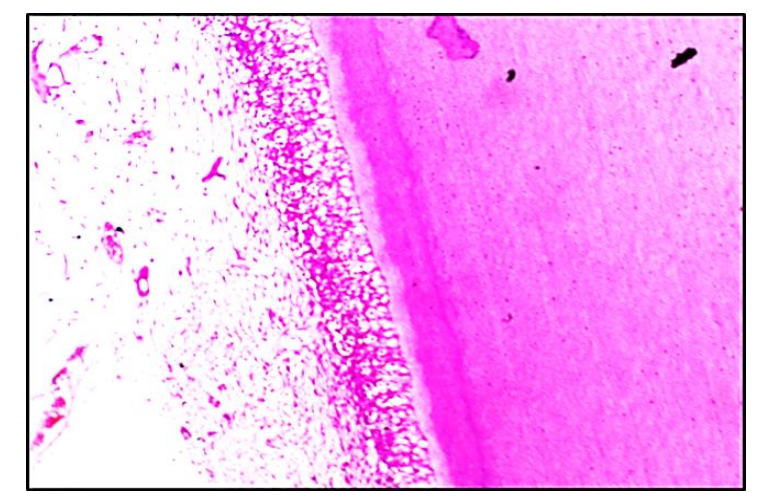

Fig. 3. Ultra Blend Plus one month; the predentin is laid down in the exposure site (H\&E X 100).

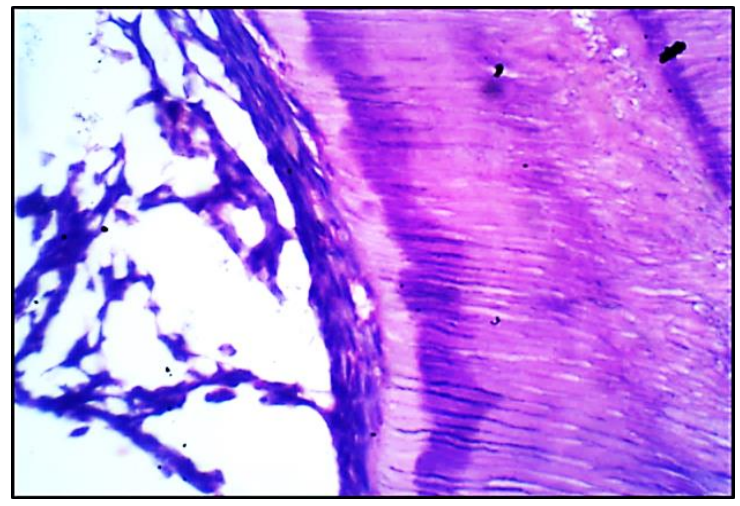

Fig. 4. L.M. Ultra Blend Plus one month showing; Flattened odontoblast like cells beneath which scattered fine fibrils extending towords inner boundry of exposure site. (H\&E X 100). 


\section{Third observational period (Three months interval)} (Fig. 6,7,8)

This period was characterized by dentin bridge formation but with differences in thickness and quality in the three tested materials.

Dycal: The superficial area directly opposite to the exposure site showed irregular bridge of hard tissue.

UBP: The superficial area directly opposite to the exposure site showed bridge of hard tissue formation, with tubular appearance. The pulp tissue reveled some calcification in thin delicate fibrous connective tissue and multiple layers of odontoblasts.

Dentin bridges appeared thicker than those observed in association with Dycal and less homogenous with an obvious continuation with the edges of the exposure site, with normal pulp morphology. The odontoblast like cells adjacent to the formed dentin bridge appeared slightly disorganized, although they faced thin ribbons of predentin containing the odontoblastic processes which were followed within the hard material of the dentin bridge.

TheraCal: An important observation here in this period was the formation of hard calcified tissues of moderate thickness related to the exposure site. A dentine bridge was observed with no tunnel defects or soft tissue inclusion. Intact multiple layers of vital odontoblasts and congested dilated vessels could be seen. A continuous homogenous bridge of dentin completely spanning the distance between the two edges of the exposure site was evident with an outstanding broad union with dentin on both sides. The odontoblasts adjacent to the formed bridge exhibited almost normal organization and they were resting adjacent to a band of predentin containing the extension of the odontoblastic processes.

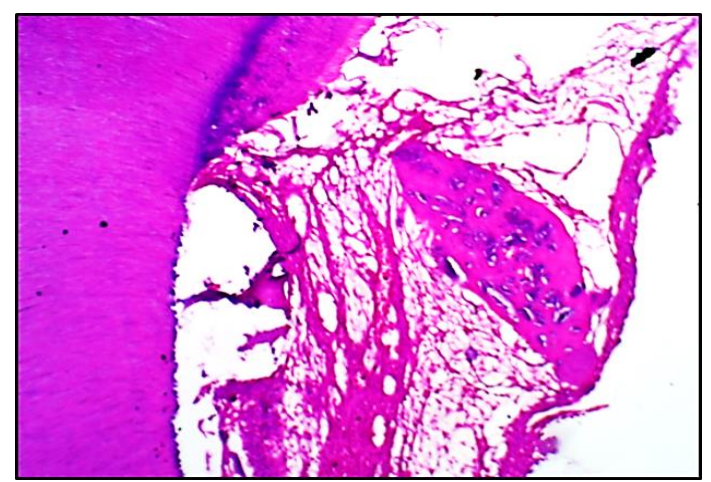

Fig. 5. L.M. Theracal group one month showing; thin irregular osteodentin bridge (H\&E X 100).

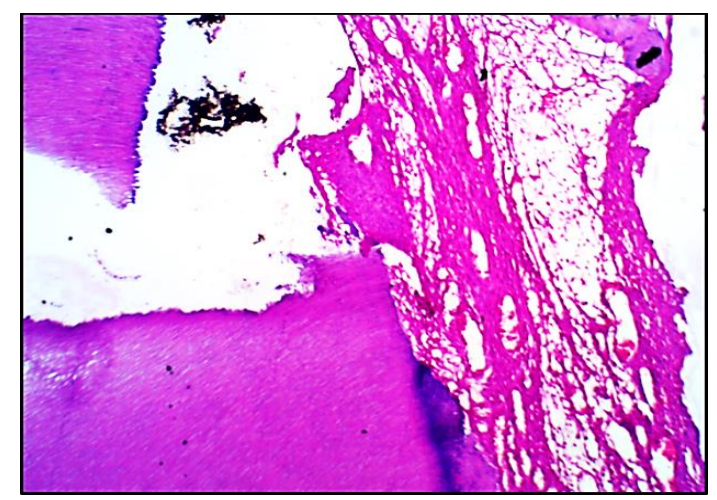

Fig. 6. L.M. High magnification Dycal three months showing; superficial area directly opposite to the exposure site showed a bridge of hard tissue formation (H\&E X 400).

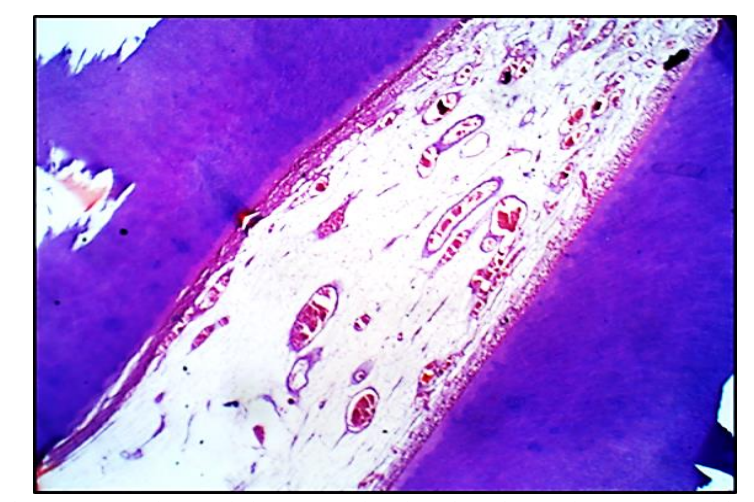

Fig. 7. L.M. Theracal three months showing; intact layer odontoblasts (H\&E X 100).

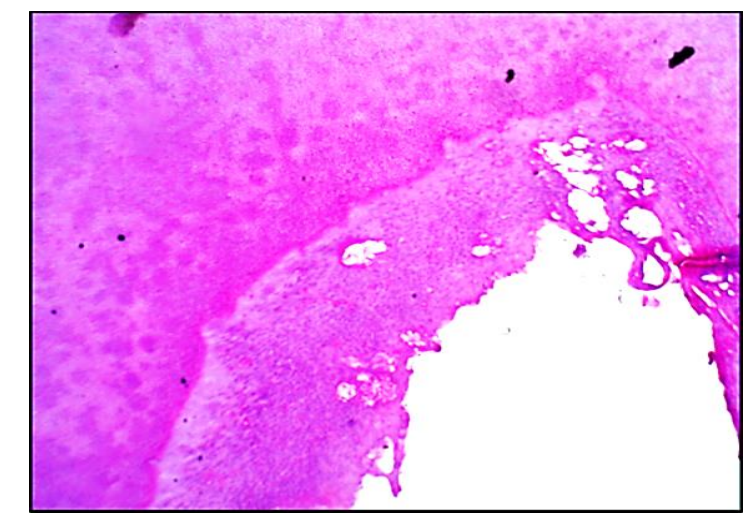

Fig. 8. L.M. Theracal three months showing; a dentine bridge with no tunnel defects or soft tissue inclusion. (H\&E X 100).

\section{DISCUSSION}

Nowika et al [9] and Suzuki et al [10] stated that clinical and histological evaluation are the gold standards for the assessment of pulp reaction to the test material; therefore, in vivo studies are required to understand pulp response when dental materials are used for direct pulp capping. The purpose of this work was to histologically evaluate the calcific barrier and odontoblast cells after direct pulp capping using three different materials: Dycal, Ultrablend plus and Theracal at three observation periods: one week, one month and three months. Negma et al [11] examined the length of time necessary for adequate postoperative follow up and suggested that 3 months period was adequate for a tentative prognosis.

In the present study, dog was the selected animal model. The synthesis of dentin in this animal is the same as in human beings as stated by El Ashry et al [12].

In the present study, the bleeding was controlled by rinsing the exposure site with sterile saline solution this method was also adapted by Al-sherbiny et al [13]. Godoy and Murray 2005 concluded that bleeding control is more important to preserve pulp vitality than is the choice of a specific direct pulp capping agent, because of evidence that pulp healing is most compromised by hemorrhagic injury [14].

In order to assess the biological capability of the tested materials, histopathological evaluation was performed to grade the success of the three materials based on: the evaluation of the success of the calcific barrier (continuity, morphology and presence of tubules). The presence of odontoblastic layer was also evaluated. At one week interval there was no reparative dentin or odontoblastic cell 
proliferation, only mesenchymal cells were seen in the interphase between capping materials and pulp tissue in all three materials. This finding was similar to that of Stefanova et al [15], who did not find evidences for reparative dentin bridge formation near by the preparation in the first/second week of the experiment.

In histologic studies, reparative dentin is commonly seen as a final zone of dentin-like matrix in association with a zone of hard tissue followed by a layer of odontoblast like cells. Thus, the therapeutic validity of a given pulp capping material might be evaluated on the basis of its ability to stimulate directly odontoblast like cell differentiation [16].

At one month interval most of our samples exhibited odontoblast like cells under the formed matrix. This is in concordance with the results of Sadek H and Abou el Nasr $\mathrm{H}$ [17]. Jalan et al [18] noticed that after pulp capping with $\mathrm{Ca}(\mathrm{OH})_{2}$, the appearance of dentine-like tissue was observed in 4 weeks after the procedure. This could be explained by Maden et al [19] who stated that $\mathrm{Ca}(\mathrm{OH})_{2}$ produces a superficial pulp necrosis then dystrophic calcification is deposited immediately beneath the granular zone, where odontoblast-like cells differentiate and organize to produce dentin.

In current study, deposition of the reparative dentin was an evident aspect observed at one month in most samples from all materials, but it was irregular and of different thickness. Such features were indicative of recovery and maintenance of the remaining pulp tissue's vitality. Pereira et al [20], stated that the presence of dentin bridge must be recognized not only as a barrier to future injuries, but also as a sign of biological recovery, represented by the odontoblast activity. Hard tissue bridge formation at 28 days was noticed by Cannon et al [21] in their study on theraCal LC was more significant than the GIC and VLC Dycal groups. Gandolfi et al [22], found that TheraCal LC release more calcium than Dycal throughout the tested period (28 days).

After three months: our study revealed the presence of odontoblast-like cell formation and early tubule development in most specimens of the Dycal, as well as UBP and TheraCal LC.

The three materials induced the formation of a dentinal bridge at its interface with the pulp tissue, however, the hard tissue induction was different with light cured materials compared to Dycal, though the difference was not statistically significant.

The success of $\mathrm{Ca}(\mathrm{OH})^{2}$ as a pulp-capping agent was most probably due to the stimulation of odontoblast activity and subsequent mineralization. Compared to the resincontaining calcium hydroxide (Ultra-Blend Plus), the resincontaining calcium silicates (TheraCal LC) presented lower toxcicity to cultured pulp cells [23].

TheraCal LC contains a hydrophilic monomer/polymer matrix, while Ultra-Blend Plus contains a hydrophobic monomer/polymer matrix. The first, allows higher calcium and hydroxide release, which are very important in protecting pulp, stimulating dentin formation, and providing antibacterial action due to high alkalinity, as explained by Chen et al. [24] and Gandolfi et al [22].

It must be noted that, rather than the pulp capping agents, the subjacent pulp tissue is responsible for the pulpal healing associated with hard tissue barrier formation.
Gandolfi et al [22], reported that TheraCal LC induced lower $\mathrm{pH}$ alkalization than the conventional self-setting Dycal did. As its solubility is lower than that of Dycal, hence, TheraCal LC may act as a scaffold for reparative dentine formation. Both TheraCal LC and UBP showed similar or lower cytotoxicity than Dycal.

Other studies indicated that the success rate for TheraCal LC was not significantly different from that for Dycal in direct pulp capping after 6-month follow-up [6], [16], [25].

TheraCal standed out as superior in most of the above studies in terms of physical and chemical properties, sealing ability, Biocompatibility and Antimicrobial properties [26], [27].

Scarano et al. [23], concluded that direct pulp capping is a worthwhile procedure that should be performed when indicated, especially in light of the $90.3 \%$ survival rate of direct pulp cap at 54 months.

\section{Conclusions}

The subjacent pulp tissue is responsible for the pulpal healing associated with hard tissue barrier formation, together with the pulp capping agents.

Gradual healing over the study period is a sign of biological acceptance of the materials used in this investigation

Theracal is superior to Dycal and UBP for pulp capping of mechanically exposed human teeth, it can be used as an effective direct pulp capping material.

\section{REFERENCES}

[1] Tomás-Catalá CJ, Collado-González M, García-Bernal D, OñateSánchez RE, Forner L, Llena C, et al. Biocompatibility of new pulpcapping materials NeoMTA plus, MTA repair HP, and biodentine on human dental pulp stem cells. Journal of endodontics. 2018; 44(1):126-32.

[2] Gopikrishna V, Pradeep G, Venkateshbabu N. Assessment of pulp vitality: a review. International Journal of Paediatric Dentistry. 2009;19(1):3-15.

[3] Cvek M. A clinical report on partial pulpotomy and capping with calcium hydroxide in permanent incisors with complicated crown fracture. Journal of endodontics. 1978; 4(8):232-7.

[4] Aguilar P, Linsuwanont P. Vital pulp therapy in vital permanent teeth with cariously exposed pulp: a systematic review. Journal of endodontics. 2011;37(5):581-7.

[5] Parolia A, Kundabala M, Rao N, Acharya S, Agrawal P, Mohan M, et al. A comparative histological analysis of human pulp following direct pulp capping with Propolis, mineral trioxide aggregate and Dycal. Australian dental journal. 2010;55(1):59-64.

[6] Hebling J, Lessa FC, Nogueira I, Carvalho RN, Costa AC.Cytotoxicity of resin-based light-cured liners. American Journal of Dentistry 2009, 22 (3): 137-42.

[7] Chaudhari WA, Jain RJ, Jadhav SK, Hegde VS, Dixit MV. Calcium ion release from four different light-cured calcium hydroxide cements. 2016; 28 (2): 114-18.

[8] Nowicka A, Lipski M, Parafiniuk M, Sporniak-Tutak K, Lichota D, Kosierkiewicz A, et al. Response of human dental pulp capped with biodentine and mineral trioxide aggregate. J Endod. 2013;39(6):74349.

[9] Nowicka A, Łagocka R, Lipski M, Parafiniuk M, Grocholewicz K, Sobolewska E, et al. Clinical and histological evaluation of direct pulp capping on human pulp tissue using a dentin adhesive system. BioMed research international 2016; (4):1-9.

[10] Suzuki M, Kato C, Kawashima S, Shinkai K. Clinical and histological study on direct pulp capping with $\mathrm{CO} 2$ laser irradiation in human teeth. Operative Dentistry. 2019;44(4):336-47.

[11] Negm AM, Hassanien EE, Abu-Seida AM, Nagy MM. Biological evaluation of a new pulp capping material developed from Portland cement. Experimental and Toxicologic Pathology. 2017;69(3):115-22. 
[12] El Ashry SH, Abu-Seida AM,Bayoumi AA, Hashema AA. Regenerative potential of immature permanent non-vital teeth following different dentin surface treatments. Experimental and Toxicologic Pathology $2016 ;(68): 181-190$

[13] Al-Sherbiny IM, Abu-Seida A, Farid M, Motawea I, Bastawy H. Histopathological pulp response of dog's teeth capped with biosealer and biodentine: An in vivo study. Saudi Endodontic Journal 2020; 10(3):226-233

[14] Godoy F, Murray P. Systemic evaluation of various haemostatic agents following local application prior to direct pulp capping. Braz J Oral Sc 2005; 4(14):791-797.

[15] Stefanova V, Tsanova S, Atanasova P, Borisov I, Chaprazov T, Tsanova M. early resultsafter direct pulp capping witht calcium silicate based material and and mieral trioxide aggregate and ER: YAG cavity preparation in dogs. Trakia Journal of Sciences 2014;12(1):331-4.

[16] Cengiz SB, Batirbaygil Y, Onur MA, Atilla P, Asan E, Altay N. Histological comparison of alendronate, calcium hydroxide and formocresol in amputated rat molar. Dent Traumatol 2005; 21(5):2818.

[17] Sadek H, Abou El Nasr H. Histologic assessment of pulp response to strontium ranelate and bisphospho-nates when compared to calcium hydroxide as direct pulp capping materials. An animal study. Egyptian Dental Journal. 2020;66(2):1403-12.

[18] Jalan AL, Warhadpande MM, Dakshindas DM. Comparison of human dental pulp response to calcium hydroxide and biodentine as direct pulp-capping agents. Journal of Conservative Dentistry: JCD, 2017, 20(2):129-133.

[19] Maden M, Orhan EO, Ertgul E F, Sengüven B. The inflammatory response of the pulp after direct capping with platelet-rich plasma and enamel matrix derivative: A controlled animal study. Open Journal of Stomatology. 2014;(4):01:8.

[20] Pereira KDF, Cruvinel RFDS, Dantas AAR, Kuga MC. Evaluation of calcium release and $\mathrm{pH}$ value of light-cured cavity liners for pulpcapping materials. Revista de Odontologia da UNESP 2018;47:205.

[21] Cannon M, Gerodias N, Vieira A, Percinoto C, Jurado R. Primate pulpal healing after exposure and TheraCal application. Journal of Clinical Pediatric Dentistry 2014;38:333-7.

[22] Gandolfi MG, Siboni F, Prati C. Chemical-physical properties of TheraCal, a novel light-curable MTA-like material for pulp capping. Int Endod J 2012;45:571-9.

[23] Scarano A, Manzon L, Di Giorgio R, Orsini G, Tripodi D, Piattelli A. Direct Capping with Four Different Materials in Humans: Histological Analysis of Odontoblast Activity. Journal of endodontics 2003;29:729-34.

[24] Chen L, Suh BI. Cytotoxicity and biocompatibility of resin-free and resin-modified direct pulp capping materials: A state-of-the-art review. Dental materials journal. 2017;36(1):1-7.

[25] Arandi NZ, Rabi T. TheraCal LC: From Biochemical and Bioactive Properties to Clinical Applications. International Journal of Dentistry 2018;2018:3484653.

[26] Kamal EM, Nabih SM, Obeid RF, Abdelhameed MA. The reparative capacity of different bioactive dental materials for direct pulp capping. Dental and medical problems 2018;55:147-52.

[27] Kunert M, Lukomska-Szymanska M. Bio-Inductive Materials in Direct and Indirect Pulp Capping-A Review Article. Materials 2020;13:1204. 